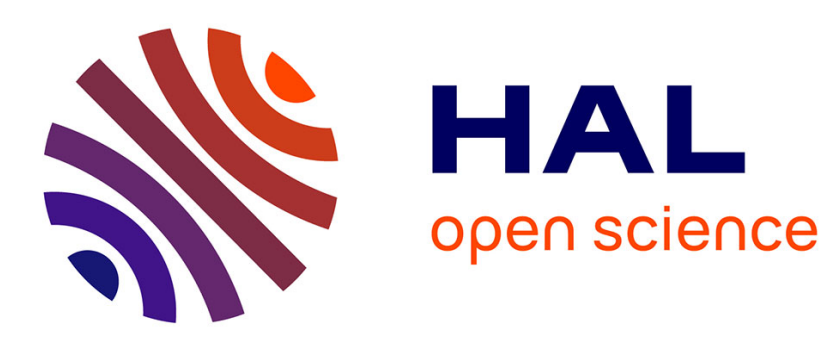

\title{
Mask Estimation For Missing Data Recognition Using Background Noise Sniffing
}

Sébastien Demange, Christophe Cerisara, Jean-Paul Haton

\section{To cite this version:}

Sébastien Demange, Christophe Cerisara, Jean-Paul Haton. Mask Estimation For Missing Data Recognition Using Background Noise Sniffing. IEEE International Conference on Acoustics, Speech, and Signal Processing - ICASSP 2006, May 2006, Toulouse/France. inria-00080562

\section{HAL Id: inria-00080562 https://hal.inria.fr/inria-00080562}

Submitted on 19 Jun 2006

HAL is a multi-disciplinary open access archive for the deposit and dissemination of scientific research documents, whether they are published or not. The documents may come from teaching and research institutions in France or abroad, or from public or private research centers.
L'archive ouverte pluridisciplinaire HAL, est destinée au dépôt et à la diffusion de documents scientifiques de niveau recherche, publiés ou non, émanant des établissements d'enseignement et de recherche français ou étrangers, des laboratoires publics ou privés. 


\title{
MASK ESTIMATION FOR MISSING DATA RECOGNITION USING BACKGROUND NOISE SNIFFING
}

\author{
Sébastien Demange, Christophe Cerisara and Jean-Paul Haton \\ LORIA - UMR 7503 \\ 54500 Vandoeuvre-les-Nancy - FRANCE \\ demangs@loria.fr, cerisara@loria.fr, jean-paul.haton@loria.fr
}

\begin{abstract}
This paper addresses the problem of spectrographic mask estimation in the context of missing data recognition. At the difference of other denoising methods, missing data recognition does not match the whole spectrum with the acoustic models, but rather considers that some time-frequency pixels are missing, i.e. corrupted by noise. Correctly estimating these "masks" is very important for missing data recognizers. We propose a new approach that exploits some $a$ priori knowledge about these masks in typical noisy environments to address this difficult challenge. The proposed mask is then obtained by combining these noise dependent masks. The combination is led by an environmental "sniffing" module that estimates the probability of being in each typical noisy condition. This missing data mask estimation procedure has been integrated in a complete missing data recognizer using bounded marginalization. Our approach is evaluated on the Aurora2 database.
\end{abstract}

\section{INTRODUCTION}

Robustness of automatic speech recognition to noise is a major challenge for nowadays state-of-the-art systems. While a number of efficient methods have been proposed to deal with quasi-stationary or slowly varying noise, very few techniques can handle non-stationary and highly variable noise. Missing Feature Theory (MFT) is such an approach, which assumes that the noise masks the speech signal in some localized spectrographic regions.

\section{A typical MFT speech recognizer is composed of two stages:}

The first step produces a mask that identifies the reliable spectrographic features (dominated by speech), and the corrupted features (dominated by noise). This mask is defined for every frame and every spectral coefficient: it is thus composed of $T \times N$ boolean values, where $T$ is the total number of frames and $N$ is the number of frequency bands at the output of the front-end. Soft masks have also been proposed [1] to encode the probability that each feature is corrupted by noise instead of the hard reliable/corrupted decision.

The second step recognizes the speech by taking into account the mask. Two different approaches can be used The first one called marginalization, marginalizes the observation likelihood of the corrupted (hidden) observations. Several variants of marginalization have been proposed; a typical one is the following:

$$
P(X \mid \Theta)=P\left(X_{r} \mid \Theta\right) \cdot \int P\left(X_{m} \mid \Theta\right) d X_{m}
$$

where $X_{r}$ and $X_{m}$ are the reliable and missing coefficients of the feature vector $X$, and $\Theta$ the acoustic models. The second one called data imputation estimates the contribution of speech in the masked observations.

A number of publications have shown that MFT significantly improves the performance of speech recognizers under noisy conditions when the masks are known a priori [2]. However, this a priori information is not available in real conditions. Hence, a major challenge is to estimate accurately the missing data mask. This paper is focused on this issue.

We propose a two step method. First, several missing data masks are computed from mask estimators trained on different $a$ priori known environments. These environment dependent masks are then combined to give the final mask. The combination is led by an environment "sniffing" module that gives the probability that the unknown environment is one of the a priori environments.

The organization of the paper is as follows. In section 2, we present an overview of some state-of-the-art mask estimation techniques. In section 3, we expose our approach. Section 4 presents the experimental setup, and section 5 summarizes results. In section 6 , some limitations of the proposed method are discussed and possible improvements are suggested. Finally, conclusions and future work are given in section 7 .

\section{RELATED WORKS}

The litterature proposes a wide range of methods that estimate the masks. While some of them are motivated by psycho-acoustical considerations, others are derived from signal processing algorithms. The most important of these methods are summarized next.

Drygajlo and El-Maliki [3] proposed to exploit spectral subtraction to estimate the missing data masks. The main drawback of this approach is that spectral subtraction works well for stationary noise, but fails to capture accurately non-stationary noise.

It was also shown that the local signal-to-noise ratio (SNR) is a good criterion to classify spectrographic features as reliable or missing. However, it is difficult to correctly estimate the local SNR since the clean speech signal is usually unknown. Nevertheless, techniques based on thresholding of an estimate of local SNR (a posteriori SNR) constituted the first attempts to automatically infer the missing data masks [4]. In this thesis Renevey showed that spectral subtraction and SNR based detections are equivalent.

Another classical criterion used to estimate the masks is the harmonicity measure, which is combined in [5] and [6] with the local SNR. 
In [7], a neural oscillator network is also proposed to compute the missing data mask.

Roweis introduced an approach based on the factorial-max vector quantization model in [8]. A set of noise and speech codebooks were combined using the masking approximation, which stated that only one codebook dominates in a given frequency band and at a given frame. The resulting masks were thus obtained by finding the sequence of noise and speech codebooks that maximized the likelihood of the noisy sentence.

A top-down procedure is presented in [9] to automatically select the most appropriate masks within a set of potential candidates derived with the previous techniques.

Seltzer proposed a Bayesian classifier to label spectrographic features [10], which does not assume any prior knowledge about the noise. While Seltzer used speech corrupted by white noise to train the classifier, Kim et al. proposed in [11] a new training method based on subbands of colored noise.

\section{COMBINATION OF ENVIRONMENT DEPENDENT MASKS}

The proposed approach relies on bayesian classification of spectrographic features into two classes: reliable and missing. We propose to train several bayesian mask estimators and to combine their classification decision. Each mask estimator is trained on a particular corrupting noise at a given SNR. An environmental "sniffing" module is used to combine these specialized mask models.

We consider a finite set $E=\left\{e_{1}, \cdots, e_{K}\right\}$ of representative environments that are known a priori. Each $e_{k}$ is characterized by its nature (car noise, street noise, ...) and by its SNR. Inspired by [12], we propose to compute the probability $p\left(e_{k} \mid y\right)$ that $e_{k}$ is the background noise of frame $y$.

The basic principle of our approach is illustrated by the graphical network of figure 1 .

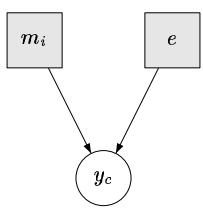

(a)

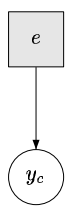

(b)
Fig. 1. Graphical representation of the dependencies in (a) our mask models and (b) our environment models. Square nodes are discrete, and greyed ones are hidden.

On this figure, $m_{i}$ is a boolean variable that represents the mask for the $i^{t h}$ coefficient of the spectral observation $y, y_{c}$ is the cepstral parametrization of $y$, and $e \in E$ is a discrete variable that represents the test environment. Two Gaussian mixture models (GMM) are considered, one for the masked and reliable coefficients given some noisy environments, and some to detect the environment. Both GMMs are defined in the cepstral domain.

The overall method can be decomposed into two steps:

\section{Step 1: Noise dependent mask estimation}

In this step, we assume that the background environment $e_{k}$ is known. The probability that the $i^{t h}$ spectral coefficient is masked is then computed:

$p\left(m_{i} \mid y_{c}, e_{k}\right)=\frac{p\left(y_{c} \mid m_{i}, e_{k}\right) \cdot p\left(m_{i} \mid e_{k}\right)}{p\left(y_{c} \mid m_{i}, e_{k}\right) \cdot p\left(m_{i} \mid e_{k}\right)+p\left(y_{c} \mid \overline{m_{i}}, e_{k}\right) \cdot p\left(\overline{m_{i}} \mid e_{k}\right)}$

where $p\left(m_{i} \mid e_{k}\right)$ and $p\left(\overline{m_{i}} \mid e_{k}\right)$ respectively represent the a priori probability that the $i^{t h}$ feature of $y$ is missing and reliable in the environment $e_{k} . p\left(y_{c} \mid m_{i}, e_{k}\right)$ and $p\left(y_{c} \mid \overline{m_{i}}, e_{k}\right)$ are modeled by two GMMs.

Step 2: Combination with environment sniffing

In this stage, the probability that $y_{c}$ is corrupted by an a-priori environment $e_{k}$ is given by:

$$
p\left(e_{k} \mid y_{c}\right)=\frac{p\left(y_{c} \mid e_{k}\right) \cdot p\left(e_{k}\right)}{\sum_{j=1}^{c a r d(E)} p\left(y_{c} \mid e_{j}\right) \cdot p\left(e_{j}\right)}
$$

We assume that all the environments have the same a priori probability: $p\left(e_{i}\right)=p\left(e_{j}\right) \forall(i, j)$. The likelihood $p\left(y_{c} \mid e_{k}\right)$ is given by a GMM. We propose to merge masks computed in the first step according to these environment probabilities. The combination scheme is a classical Bayesian derivation :

$$
p\left(m_{i} \mid y_{c}\right)=\sum_{k=1}^{\operatorname{card}(E)} p\left(m_{i} \mid y_{c}, e_{k}\right) \cdot p\left(e_{k} \mid y_{c}\right)
$$

Finally, masks can be designed either in a soft fashion by taking $p\left(m_{i} \mid y_{c}\right)$ as the mask value, or in a hard fashion by thresholding this probability :

$$
m_{i}=\left(p\left(m_{i} \mid y_{c}\right) \geq \delta\right)
$$

where $\delta$ is a threshold in the interval $[0,1]$. The complete architecture of our mask estimator is presented in figure 2 .

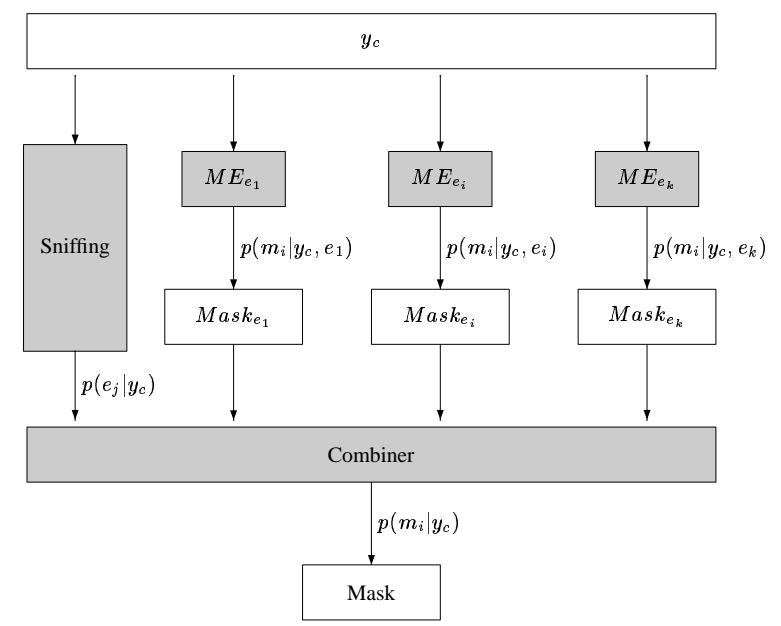

Fig. 2. Our missing data mask estimator architecture. " $M E_{e_{k}}$ " is a mask estimator trained on the a-priori known environment $e_{k}$. " $p\left(m_{i} \mid y, e_{k}\right)$ " is the probability that the $i^{t h}$ coefficient of $y$ is missing when $e_{k}$ is supposed to be the corrupting environment. Each mask estimator $M E_{e_{k}}$ provides an environment dependent mask "Mask $e_{e_{k}}$ " from $p\left(m_{i} \mid y_{c}, e_{k}\right)$. These masks are combined according to the environment sniffing module that gives the probability of being in each a-priori noisy condition. 

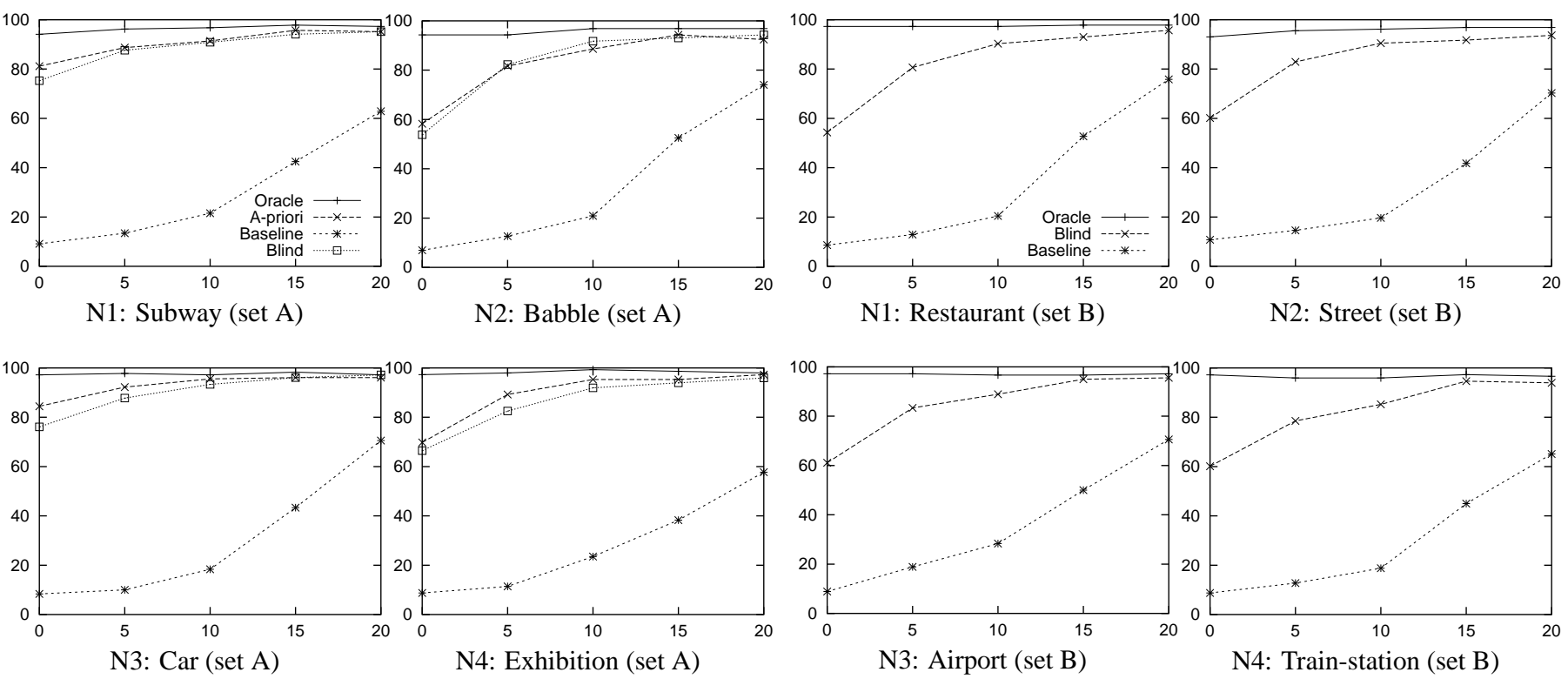

Fig. 3. Recognition accuracy for test set A of Aurora2 database.

\section{EXPERIMENTAL SETUP}

All the experiments reported in this paper are based on the Aurora2 database speaker independent connected digit recognition task [13].

\subsection{Models training}

The environment sniffing and mask models are trained on 800 sentences of the Aurora2 test sets and tested on 50 other sentences. Two parametrizations of the signal are used. The first one is the classical set of $13 \mathrm{Mel}$ Cepstral Coefficients (MFCC) supplemented with their temporal derivatives, which gives a 26 dimensional feature vector. This parametrization is used to estimate the masks and the environments. The second one is the classical $32 \mathrm{Mel}$ spectral coefficients with a cuberoot-compression. It is used for speech recognition and digits models training. The 28 environments $(4$ noise at 7 SNRs) of the training corpus of Aurora2 define our set of a-priori known environments. Two GMMs with 256 Gaussians are trained on each of these environments $e_{k}$, for each frequency band $i$. They respectively model the probability density functions $p\left(y_{c} \mid m_{i}, e_{k}\right)$ and $p\left(y_{c} \mid \overline{m_{i}}, e_{k}\right)$. In addition, a GMM with 256 Gaussians is trained on all the observations of a given environment. It models the probability density functions $p\left(e_{k} \mid y_{c}\right)$.

The a-priori probability that a spectrographic feature is missing $p\left(m_{i} \mid e_{k}\right)$ is a scalar value for each frequency band of each $a$ priori environment. Whole word digit models are trained on the Aurora2 clean speech training set. These models are standard 16 states HMMs with 7-component mixtures. The task grammar forces every sentence to begin and end by a silence.

\subsection{Missing data recognition}

Soft bounded marginalization is used during recognition. Any spectral coefficient is considered as missing when the local SNR is below $0 \mathrm{~dB}$, and reliable otherwise. Thanks to cuberoot-compression, this

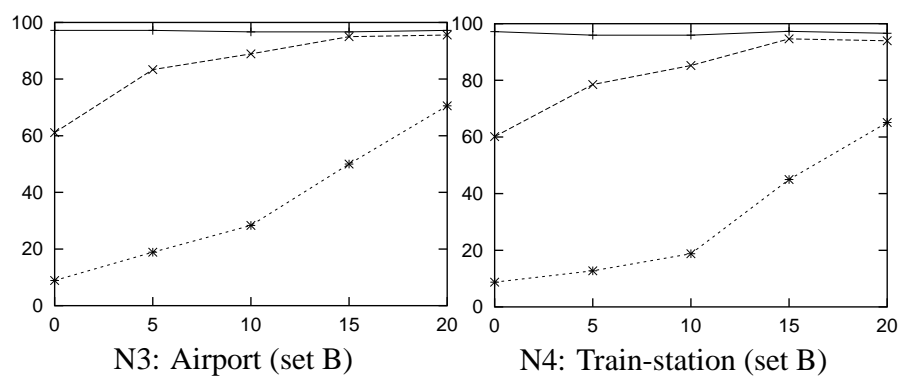

Fig. 4. Recognition accuracy for test set B of Aurora2 database.

implies that the speech contribution $x_{i}$ of any observed missing coefficient $y_{i}$ is in the interval:

$$
0 \leq x_{i} \leq \sqrt[3]{y_{i}^{3} / 2}
$$

Conversely, the speech contribution $x_{i}$ of any observed reliable coefficient $y_{i}$ is in the interval:

$$
\sqrt[3]{y_{i}^{3} / 2} \leq x_{i} \leq y_{i}
$$

For simplicity, we set $y_{i, s n r 0}=\sqrt[3]{y_{i}^{3} / 2}$. Hence, the emission probability of a particular feature vector $y$ is expressed as follow:

$$
\begin{aligned}
p(y \mid \Theta) & =\prod_{i}\left\{\frac{p\left(m_{i} \mid y_{c}\right)}{y_{i, s n r 0}} \cdot \int_{0}^{y_{i, s n r 0}} p\left(x_{i} \mid \Theta\right) d x_{i}\right. \\
& \left.+\frac{p\left(\overline{m_{i}} \mid y_{c}\right)}{y_{i}-y_{i, s n r 0}} \cdot \int_{y_{i, s n r 0}}^{y_{i}} p\left(x_{i} \mid \Theta\right) d x_{i}\right\}
\end{aligned}
$$

where $\Theta$ reflects state parameters. This soft bounded marginalization was introduced in [14].

\section{EXPERIMENTAL RESULTS}

\subsection{Validation of the approach with known environments}

Figure 3 shows the recognition accuracy of the proposed approach on test set A. The "Baseline" and "Oracle" systems respectively give the recognition accuracy without masking any data, and with the optimal masks computed from the true SNR.

The "A-priori" system presents the results when the environment is known a-priori. We can observe that the proposed approach gives quite good recognition results, comparable with the other missing data systems reported in the litterature.

The "Blind" system does not assume any knowledge about the environment. The results given by the "A-priori" and "Blind" systems are quite close, which validates the environment sniffing module. The average accuracy of the blind system on test set A is $81.47 \%$. 


\subsection{Tests with unknown environments}

Figure 4 shows the recognition rates obtained on test set $B$, where the noise types are different from those used to train the mask and environment models. Only the blind system can be represented on these figures.

The average recognition accuracy of the blind system on test set $\mathrm{B}$ is $77.38 \%$. We can observe a slight decrease of the performances when there is a mismatch between the training and test environments. However, the recognition results are still comparable with the other state-of-the-art missing data recognition approaches. This confirms the potential of the proposed method.

\section{DISCUSSION}

The proposed approach is based on a set of known "typical" noisy environments. This raises two important issues: how can we deal with unknown environments that are completely different from the training conditions, and how large can be, or should be, the set of training environments.

Regarding the first issue, we are currently investigating some solutions to adapt the models to a new environment that do not belong to the training database.

About the second issue, a very large noisy database is probably not the best option, as the confusion between the GMMs might increase with the number of different environments. In this work, we considered 4 noises and 7 SNRs, which lead to 28 training conditions. The results show that such a number of environments is not excessive. If we want to use this system in many possible conditions, a solution may be to cluster all these environments hierarchically, and to use external algorithms to pre-select a small subset of them, before applying our combination method. An alternative to reduce the number of different environments would be to use a "canonical" noise database, which would represent concisely a wide variety of noises, as it is done in the eigenvoices method for speaker voices. Adaptation can further be considered to fine-tune the models to specific test conditions.

\section{CONCLUSIONS AND FUTURE WORK}

In this paper we have presented a new missing data mask estimator. Each spectrographic feature is classified as reliable or missing using an environment dependent model. The resulting masks, one for each candidate environment, are then combined. The noise dependent masks combination is led by an environment sniffing module that gives the probability to be in one of the training environments and at a given SNR. The current mask estimator defines a baseline and shall be modified in several aspects to improve its accuracy. In particular, the classical MFCC signal parametrization is used in our system, but better parameters can be used to distinguish reliable features from missing ones. Those proposed by Seltzer are good examples. Moreover, we plan to integrate temporal dependencies in our models, either in the form of HMMs, or with context dependent models similar to the n-gram models used in language modeling. Finally, different combination schemes can be studied.

\section{ACKNOWLEDGEMENTS}

This work was supported by the HIWIRE (Human Input That Works In Real Environments) project consortium.

\section{REFERENCES}

[1] Andrew Morris, Jon Barker, and Herv Bourlard, "From missing data to maybe useful data: soft data modelling for noise robust ASR," in Proc. WISP-01, Stratford-upon-Avon, England, April 2001, pp. 153-164.

[2] Jon Barker, Phil Green, and Martin Cooke, "Linking auditory scene analysis and robust ASR by missing data techniques," in Proc. WISP, Stratford-upon-Avon, England, April 2-3 2001, pp. 295-307.

[3] Andrzej Drygajlo and Mounir El-Maliki, "Speaker verification in noisy environments with combined spectral subtraction and missing feature theory," in ICASSP, Seattle, 1998, pp. 121124.

[4] Philippe Renevey, Speech recognition in noisy conditions using missing feature approach, Ph.D. thesis, Ecole Polytechnique Fédérale de Lausanne, 2001.

[5] Jon Barker, Martin Cooke, and Phil Green, "Robust asr based on clean speech models: an evaluation of missing data techniques for connected digit recognition in noise," in Proc. EUROSPEECH, Denmark, September 2001.

[6] Hugo Van hamme, "Robust speech recognition using cepstral domain missing data techniques and noisy masks," in ICASSP, Montreal, Quebec, Canada, 2004, vol. 1, pp. 213-216.

[7] G. J. Brown, J. Barker, and D. L. Wang, "A neural oscillator sound separator for missing data speech recognition," in Proc. IJCNN-01, Washington, DC, USA, 2001, vol. 4, pp. 2907-2912.

[8] Sam T. Roweis, "Factorial models and refiltering for speech separation and denoising," in Proc. EUROSPEECH, Geneva, Switzerland, 2003, pp. 1009-1012.

[9] Jon Baker, Martin Cooke, and Daniel P.W. Ellis, "Decoding speech in the presence of other sources," Speech Communication, vol. 45, no. 1, pp. 5-25, January 2005.

[10] Michael L. Seltzer, "Automatic detection of corrupt spectrographic features for robust speech recognition," M.S. thesis, Departement of Electrical and Computer Engineering, Carnegie Mellon University, 2000.

[11] Wooil Kim, Richard M. Stern, and Hanseok Ko, "Environment-independent mask estimation for missingfeature reconstruction," in Proc. INTERSPEECH, Lisbon, Portugal, September 2005, pp. 2637-2640.

[12] Murat Akbacak and John H.L. Hansen, "Environmental sniffing : robust digit recognition for an in-vehicle environment," in Proc. EUROSPEECH, Geneva, Switzerland, September 2003, pp. 1-4.

[13] D. Pearce and H.-G. Hirsch, "The aurora experimental framework for the performance evaluation of speech recognition systems under noisy conditions," in Proc. ICSLP, Beijing, China, October 2000, vol. 4, pp. 29-32.

[14] Andrew Morris, "Data utility modelling for mismatch reduction," in Proc. CRAC (workshop on Consistent \& Reliable Acoustic Cues for sound analysis), Aalborg, Denmark, September 2001. 